\title{
Computing with Highly Mixed States
}

\section{[Extended Abstract]}

\author{
Andris Ambainis* \\ Computer Science Division \\ U. C. Berkeley \\ Berkeley CA 94720
}

\author{
Leonard J. Schulman ${ }^{\dagger}$ \\ College of Computing \\ Georgia Tech \\ Atlanta GA 30332-0280
}

\author{
Umesh V. Vazirani ${ }^{\ddagger}$ \\ Computer Science Division \\ U. C. Berkeley \\ Berkeley CA 94720
}

\begin{abstract}
We consider quantum computing in the one-qubit model where the starting state of a quantum computer consists of $k$ qubits in a pure state and $n-k$ qubits in a maximally mixed state. We ask the following question: is there a general method for simulating an arbitrary $m$-qubit pure state quantum computation by a quantum computation in the $k$ qubit model? We show that, under certain constraints, this is impossible, unless $m=O(k+\log n)$.
\end{abstract}

\section{INTRODUCTION}

Ideally, a quantum computation is a sequence of local unitary transformations applied to a register of qubits which are initially in the state $\left|0^{n}\right\rangle$; followed by a measurement. Initializing the state of the quantum register is the biggest challenge in NMR quantum computing (which is perhaps the most advanced technology in terms of the scale of experiments performed to date [2]). The difficulty is that the register is actually initially in (approximately) the binomial distribution over pure states $|x\rangle$, in which each qubit is independently in the state $|0\rangle$ with probability $\frac{1+\epsilon}{2}$; the currently achievable polarization $\epsilon$ is quite small. There are currently two ways of implementing quantum computation in this technology. The first is used in current experiments 5. 3, but does not scale beyond several qubits - the output signal decreases exponentially in the number of qubits in the quantum register.1 The second does scale, but is not feasible at the currently achievable values of polarization in

\footnotetext{
${ }^{*}$ Supported in part by a U.C. Berkeley Graduate Fellowship and NSF grant CCR-9800024. ambainis@cs.berkeley.edu ${ }^{\dagger}$ Supported in part by NSF CAREER grant CCR-9876172. schulman@cc.gatech.edu

${ }^{\ddagger}$ Supported in part by NSF grant CCR-9800024. vazirani@cs.berkeley.edu

1 The exponential decay in signal to noise ratio in any scheme that embeds virtual pure states on an $n$-qubit quantum computer with one clean qubit is unavoidable, due to the result [9].
}

liquid NMR 11. An intriguing third possibility was raised in [-]. Suppose we start with one qubit in the pure state $|0\rangle$ in tensor product with $n-1$ qubits in a maximally mixed state (i.e. in a uniform distribution over basis states $|x\rangle$ ). Is it possible to simulate general quantum computation by effecting a sequence of elementary quantum operations on this register? If the answer were affirmative, this would yield a procedure that would both scale and be currently feasible using the scalable initialization procedure to convert the initial binomial state to a state where the last $n-1$ qubits are maximally mixed and the first bit has high polarization (the strength of the output signal is now proportional to this polarization). This is the question we focus on in this paper. It is easy to see that if all $n$ qubits are in the maximally mixed state then no computation is possible. This is because applying any unitary transformation to this mixture leaves it invariant. This simple argument stands in striking contrast to the difficulty of the seemingly very similar case, in which just a single qubit is in a pure state, while all the others are maximally mixed. Since the initial state of the register is completely specified, the only real input in this model is the sequence of elementary quantum operations. So, given a quantum circuit $C$ which we would like to simulate on an input $x$, we wish to know whether there is a sequence of elementary quantum operations on the $n$ qubit register, which first prepares a quantum (mixed) state which encodes $x$, and then simulates $C$ on it. Of course, we will require that these mixed state encodings of basis states $x$ be distinguishable by some measurement with non-trivial probability.

Our main result shows that the above is impossible unless $|x| \in O(\log n)$, showing that the simulation is no more efficient than an exhaustive classical calculation. The technique used to show this uses some information about the representations of the symmetric group. The appendix 7 provides some necessary notions from representation theory.

We also show that using a 3-bit register it is possible to compute every language in NC1. This should give some indication of why the impossibility result is so much harder than for the case when all $n$ qubits are maximally mixed.

\section{NC1}

We begin by showing that in this model, even using a 3-bit register, we can compute every language in $\mathrm{NC1}$.

Recall that the initial state of the register is a uniform distribution over the four 3-bit strings starting with a 0 . In our simulation of $\mathrm{NC1}$, all our operations will simply permute basis states. 
Proposition 1. A 3-bit quantum computer initialized with one clean qubit can recognize every language in NC1.

Our simulation is based on Barrington's result that NC1 can be simulated by a width 5 permutation branching program [1]. The main idea is quite simple: let the 5 states of the permutation branching program be represented by the states $|000\rangle$ through $|100\rangle$ of the 3 -qubit register. Without loss of generality assume that the permutation branching program accepts if the permutation it effects is the identity, and rejects if the permutation it effects is the transposition $(000,100)$. It is easy to simulate the permutation branching program by a sequence of elementary quantum operations. Now if we measure the first qubit in the register, then in the case that the permutation branching program accepts - i.e. the permutation effected is the identity - then measuring the first qubit in the register yields a 0 with probability 1 . On the other hand, if the permutation branching program rejects, then measuring the first qubit in the register yields a 1 with probability $1 / 4$.

It is illuminating to try to extend this simulation to QNC1. First notice that in Barrington's procedure for simulating $\mathrm{NC} 1$, each wire in the $\mathrm{NC} 1$ circuit is simulated at some stage in the branching program. In the case of a QNC1 circuit, the state of a wire is given by a qubit, which is, in general, entangled with the qubits carried by the other wires in the circuit. Therefore the state of this wire cannot be expressed in isolation, and there appears to be no alternative to creating that entangled state as part of any simulation. Thus the entire approach breaks down. One way to carry out such a construction, might be to apply a superposition of operations at each step: this extends the state space of the quantum computer and effectively provides many more clean qubits, making the model meaningless. Moreover all proposed implementations of quantum computation involve a classical, time-varying sequence of operations, applied to a quantum register. Since the control is classical, in any oblivious simulation the entangled quantum state of the simulated circuit must be encoded within the quantum register.

\section{LIMIT ON COMPUTABILITY}

We are given a quantum computer with an $n$ qubit register, with one bit initialized to $|0\rangle$ and the rest of the $n-1$ qubits in a maximally mixed state. We would like to simulate an $m$ qubit quantum circuit $C$ on input string $x$ using this model. If we wish to do an oblivious simulation, as sketched in the previous section, we must encode an arbitrary $m$ qubit state into the uninitialized $n$ qubit register. To do so, it is sufficient to consider the $2^{m}$ basis states of the $m$ wires, and encode them as distinguishable states of the uninitialized $n$ qubit register (for this to be an efficient encoding, we should have $n \in O(\operatorname{poly}(m)))$. The states must be distinguishable in the following sense: since we can prepare several copies of any state by repeating the simulation, we only require that there be a sequence of measurements on $O(\operatorname{poly}(n))$ many copies of the state, that (with high probability) uniquely identify the state. Indeed, it is possible to do this with $n=m$, as follows: take the subspaces, spanned by the basis vectors in the sets $A_{b}=\left\{x \in\{0,1\}^{n}: x \cdot b=0 \bmod 2\right\}$ for $b \in\{0,1\}^{n}$.

However, to perform an oblivious simulation, the encoding must satisfy another property - permutability. The quantum circuit $C$ might carry out any unitary operation on its quantum state, and in particular an arbitrary permutation on its classical states. Again it is not hard to demonstrate an efficient encoding that satisfies this permutability condition, without distinguishability: take the subspaces spanned by the basis vectors in the sets $A_{b}=\left\{x=\left(x_{1} \ldots x_{n}\right) \in\{0,1\}^{n}\right.$ : $x_{1}=0$ or $\left.\left(x_{2} \ldots x_{n}\right)=b\right\}$ for $b \in\{0,1\}^{n-1}$.

However, it is not possible to construct an efficient encoding that satisfies both conditions simultaneously. This is the content of the following theorem.

Let $M=2^{m}$ be the total number of basis states of the ideal quantum computer which is being simulated. Note that each $X \in \mathcal{X}$ encoding one of these, is a subspace of dimension $2^{n-1}$ within the Hilbert space $\mathbb{C}^{2^{n}}$ of the computer. If the computer has $k$ clean qubits, then $X$ is of dimension $2^{n-k}$.

THEOREM 2. Suppose that computations on $m$ qubits can be obliviously simulated in an $n$-qubit, $k$-clean-qubit computer in our model, and that $\operatorname{dim}(X \cap Y) / \operatorname{dim}(X)<1-$ $\frac{1}{\text { poly }(m)}$ for every pair of input encodings $X, Y \in \mathcal{X}$. Then $m \leq(2 k+\log n)(1+o(1))$.

This incidentally implies that the computation of an $n$-qubit, $k$-clean-qubit computer can be simulated by a classical computer with a poly $\left(n 2^{k}\right)$ computational overhead.

It may be illuminating to consider a simpler, classical analogue of our problem. A classical circuit (taking inputs in $\{0,1\}^{n}$ ) composed of reversible gates executes a permutation of $\{0,1\}^{n}$. The analogous problem (just considering the case $k=1$ ) is that we can only represent inputs as uniform probability distributions over a set of half the elements of $\{0,1\}^{n}$. (In the quantum case this corresponds to axis-parallel subspaces of dimension $2^{n-1}$.) The question is, what is the largest number of such subsets (probability distributions) which such a circuit can permute at will. It is also essential that the probability distributions be readily distinguishable by sampling, in other words the subsets must have small intersection. It is possible (though we omit it in this extended abstract) to provide a strictly combinatorial argument expressing the fact that this task is impossible for more than poly $(n)$ subsets, because of the tension between the two requirements (permutability and distinguishability). The large size of the subsets means that we have far more constraints than we have degrees of freedom. The combinatorial argument shows that if the requirement of full permutability is imposed, and we have a superpolynomial (in $n$ ) number of subsets, then the symmetric difference of every two sets must be a vanishing fraction of the size of the sets. The two types of sets $\left\{A_{b}\right\}$ described above, however, separately achieve distinguishability and permutability.

In the quantum case we have arbitrary subspaces in place of "subsets" (or correspondingly axis-parallel subspaces). And the circuit of course can perform not just permutations of the basis, but general unitary operations. In sharp contrast with the classical case, two subspaces of half the dimensionality of the space typically will not intersect. Nevertheless, the large dimension of the subspaces imposes strict constraints on an operator which must permute them; the difficulty is in formulating the incompatibility of these requirements when the number of subspaces is large and the subspaces are required to be very distinct.

Proof:

By assumption, there are unitary operators (each corresponding to some sequence of steps in the computer) per- 
muting $\mathcal{X}$ in all ways. Let $f_{\pi}$ be the unitary operator corresponding to a permutation $\pi \in S_{M}$. If we have $f_{\pi \sigma}=f_{\pi} f_{\sigma}$ for all $\pi$ and $\sigma \in S_{M}$, then the operators $f_{\pi}$ form a representation of $S_{M}$ and we can apply the representation theory of the symmetric group.

Actually, the situation is slightly more complicated.

Let $U$ be the unitary group on $\mathbb{C}^{2^{n}}$. Let $H$ be the subgroup of $U$ acting on $\mathcal{X}$, i.e. carrying any $X \in \mathcal{X}$ to some $Y \in \mathcal{X}$. Let $G$ be the subgroup of $H$ that fixes all of $\mathcal{X}$; thus $G$ is normal in $H$.

It is apparent that $H / G \cong S_{M}$, but although this means that $H$ can permute the subspaces $\mathcal{X}$ in arbitrary ways, it is different from saying that there is a subgroup of $H$ isomorphic to $S_{M}$ (or in other words that we can pick elements of $H$ so as to have these operators compose properly).

\section{PROOF OF THEOREM 2: THE SIMPLE CASE}

First, we show how to prove Theorem 1 if we can select transformations $f_{\pi}$ so that they form a representation $\left(f_{\pi} f_{\sigma}=\right.$ $\left.f_{\pi \sigma}\right)$. The more general case will be handled in the next section. Appendix (section 7) explains the notions of representation theory used in this and the next section.

We show that every pair $X, Y \in \hat{\mathcal{X}}$ have a substantial intersection. Consider the decomposition of $\mathbb{C}^{2^{n}}$ into irreducible representations $\rho_{1} \oplus \ldots \oplus \rho_{k}$. Let $N=2^{n}$.

LEMMA 3. Either the first row or the first column of the Young diagram of each $\rho_{i}$ is of length more than $M-c n$.

Proof:

This results follows from a theorem by Rasala [10]:

THEOREM 4. [10, $p p .151-152]$

1. Let $A \leq M / 2$ and $\rho$ be an irreducible representation of $S_{M}$ such that the first row of the Young diagram of $\rho$ is of length exactly $M-A$. Then,

$$
\operatorname{dim} \rho \geq \varphi_{A}(M)
$$

where $\varphi_{A}(M)=\left(\begin{array}{c}M \\ A\end{array}\right)-\left(\begin{array}{c}M \\ A-1\end{array}\right)=\frac{M-2 A-1}{M-A-1}\left(\begin{array}{c}M \\ A\end{array}\right)$ is the dimension of the irreducible representation corresponding to the partition $(M-A, A)$.

2. If $\rho$ is an irreducible representation with both the first row and the first column of length at most $M / 2$, then

$$
\operatorname{dim} \rho \geq \varphi_{\lfloor M / 2\rfloor}(M) .
$$

This theorem means that any representation with the first row of the Young diagram having length at most $M-k$, $k \leq M / 2$ has dimension at least

$$
\min _{B: A \leq B \leq M / 2} \varphi_{B}(M) .
$$

Simple algebra shows that this expression is minimized by $B=A$ if $A \leq M / 2-c \sqrt{M}$ for some constant $c$ and $B=$ $\lfloor M / 2\rfloor$ if $A>M / 2-c \sqrt{M}$.

To deduce our lemma, assume that the Young diagram of an irreducible representation of $S_{M}$ has both first row and

${ }^{2}$ In the second case, the lowest-dimensional representation actually has the first row less than $M-A$. It is quite surprsing because, in most cases, removing a square from the first row of a Young diagram and adding a square somewhere else increases the dimension. column of length at most $M-A$. We show that $N \geq 2^{A}$. Consider two cases:

Case 1: $A \geq M / 2-c \sqrt{M}$.

Notice that $A \leq M-\sqrt{M}$ because otherwise the Young diagram would fit into a square with a side less than $\sqrt{M}$ and area less than $M$. Theorem 1 implies that

$\operatorname{dim} \rho \geq \varphi_{\lfloor M / 2\rfloor}(M)=\Omega\left(\frac{2^{M}}{M \sqrt{M}}\right)=2^{M-\frac{3 \log M}{2}-O(1)}>2^{A}$.

Case 2: $A \leq M / 2-c \sqrt{M}$. Then,

$$
\begin{gathered}
\operatorname{dim} \rho \geq \varphi_{A}(M)=\frac{M-2 A+1}{M-A+1}\left(\begin{array}{c}
M \\
A
\end{array}\right) \geq \frac{1}{M}\left(\frac{M}{A}\right)^{A} \\
=\frac{1}{M}\left(\frac{M}{2 A}\right)^{A} 2^{A} \geq 2^{A} .
\end{gathered}
$$

Another lower bound on the longest row or column of a low dimension representation (for a different range of parameters) was given by Mischenko[ [.

Next consider the stabilizer of $X$ in $S_{M}$, which is isomorphic to $S_{M-1}$, and which we will denote $S_{M-1}^{X}$. $X$ decomposes into irreducible representations $V_{1}, \ldots V_{\ell}$ of $S_{M-1}^{X}$. $V_{1}$ is carried by $S_{M}$ into each $Y \in \hat{\mathcal{X}}$, and all these copies of $V_{1}$ are contained within some irreducible $W$ of $S_{M}$ in $\mathbb{C}^{2^{n}}$.

By the previous lemma, the Young diagram of $W$ has a long first column or row. We use the following fact from the representation theory of the symmetric group: when we restrict an irreducible representation $0 \rho_{\lambda}$ of $S_{M}$ of shape $\lambda$ to a subgroup $S_{M-1} \subseteq S_{M}$, it decomposes into irreducibles of $S_{M-1}$ in the following way:

$$
\rho=\bigoplus_{\lambda^{-}} \rho_{\lambda^{-}}
$$

where $\lambda^{-}$ranges over all shapes of size $M-1$ that can be obtained by deleting an "inside corner" from $\lambda$. (An inside corner is simply a point of the shape whose deletion leaves a legal shape.)

We now use:

Lemma 5. Suppose the shape $\lambda$ has a first row (column) of length $|\lambda|-\ell$ for $\ell<|\lambda| / 2$. Let $\lambda_{1}$ denote the " $\lambda$ " " obtained by deleting the last element of the first row (column). Then $\operatorname{dim}\left(\rho_{\lambda_{1}}\right) \geq \frac{|\lambda|-2 \ell}{|\lambda|} \operatorname{dim}\left(\rho_{\lambda}\right)$.

Proof: Consider the ratio $\frac{\operatorname{dim} \rho_{\lambda_{1}}}{\operatorname{dim} \rho_{\lambda}}=\frac{1}{|\lambda|} \frac{\prod_{x \in \lambda}|x|}{\prod_{x \in \lambda_{1}}|x|}$. In the last ratio, points $x$ outside of the last row (column) appear identically in the numerator and denominator. Moreover for each $x$ in the first row (column) in the numerator other than the very last point (which contributes a factor of 1 in the numerator and is absent in the denominator), the ratio between its contributions in the numerator and denominator is $\frac{|x|}{|x|-1}$. Just examining the $|\lambda|-2 \ell-1$ points of the first row furthest from the upper-left corner (and excepting the last point), we obtain a lower bound on these contributions of $\prod_{i=1}^{|\lambda|-2 \ell-1} \frac{i+1}{i}=|\lambda|-2 \ell$. Overall therefore $\frac{\operatorname{dim} \rho_{\lambda_{1}}}{\operatorname{dim} \rho_{\lambda}} \geq \frac{|\lambda|-2 \ell}{|\lambda|}$.

Lemma 6. Let $f_{\pi}$ be an $N$-dimensional representation of $S_{M}$ that acts as a permutation representation on a collection 
of $M$ subspaces $\hat{\mathcal{X}}$. Then, for any $X, Y \in \hat{\mathcal{X}}$,

$$
\operatorname{dim} X-\operatorname{dim} X \cap Y \leq \frac{2 c n}{M} N .
$$

(We write $n=\lg N$ and $m=\lg M$.)

Proof: We decompose $f_{\pi}$ into irreducible representations. Let $W$ be one of these irreducible representations. We show that $\operatorname{dim} X \cap W-\operatorname{dim} X \cap Y \cap W \leq \frac{2 c n}{M} \operatorname{dim} W$.

We look at $W$ as a representation of $S_{M-1}^{X}$. Let $V$ be the highest dimensional irreducible representation of $S_{M-1}^{X}$ within $W$. If $M<2 c n$ then the assertion is trivial. Otherwise $M-c n>M / 2$ and the hypothesis of lemma 5 is satisfied, implying that $\operatorname{dim} V \geq \frac{M-2 c n}{M} \operatorname{dim} W$. Consider two cases:

Case 1: $V \subseteq X$.

Take $\pi \in S_{M}$ such that $Y=f_{\pi}(X)$. Then, $Y \cap W=$ $f_{\pi}(X \cap W)$. Therefore, $\operatorname{dim}(Y \cap W)=\operatorname{dim}(X \cap W) \geq$ $\operatorname{dim} V \geq \frac{M-2 c n}{M} \operatorname{dim} W$ and

$$
\operatorname{dim} X \cap W-\operatorname{dim} X \cap Y \cap W \leq \frac{2 c n}{M} \operatorname{dim} W .
$$

Case 2: $V \nsubseteq X$.

Then, $V \cap X=0$ because $V \cap X$ is invariant under $S_{M-1}^{X}$ and $V$ is irreducible. $V \cap X=0$ implies

$$
\operatorname{dim} V+\operatorname{dim} X \cap W \leq \operatorname{dim} W .
$$

Together with $\operatorname{dim} V \geq \frac{M-2 c n}{M} \operatorname{dim} W$, this implies

$\operatorname{dim} X \cap W-\operatorname{dim} X \cap Y \cap W \leq \operatorname{dim} X \cap W \leq \frac{2 c n}{M} \operatorname{dim} W$.

The lemma follows by summation over all irreducible $W$.

If $f_{\pi}$ form a representation, Lemma almost immediately implies Theorem 2. Namely, we have

$$
\begin{gathered}
\frac{\operatorname{dim} X \cap Y}{\operatorname{dim} X}=\frac{\operatorname{dim} X-(\operatorname{dim} X-\operatorname{dim} X \cap Y)}{\operatorname{dim} X} \\
\geq \frac{2^{n-k}-\frac{2 c n}{M} 2^{n}}{2^{n-k}}=1-\frac{2^{k+1} c n}{M} .
\end{gathered}
$$

If this is at most $1-\frac{1}{\operatorname{poly}(m)}$, then $m \leq(k+\log n)(1+o(1))$.

\section{PROOF OF THEOREM 2: THE DIFFI- CULT CASE}

\subsection{Proof outline}

Next, we deal with the case when $f_{\pi} f_{\sigma} \neq f_{\pi \sigma}$ for some $\pi$ and $\sigma \in S_{M}$. Let $G$ be the group of transformations that map every subspace $X \in \hat{\mathcal{X}}$ to itself. Then, $f_{\pi} f_{\sigma} f_{\pi \sigma}^{-1}$ is an element of $G$ for any $\pi, \sigma \in S_{M}$. We would like to modify $f$ so that this element becomes identity for all $\pi$ and $\sigma \in S_{M}$. Then, $f_{\pi} f_{\sigma}=f_{\pi \sigma}$, i.e., $f_{\pi}$ would form a representation of $S_{M}$ and we would be able to analyse this representation similarly to the previous section.

To achieve this, we look at $\mathbb{C}^{2^{n}}$ as a representation of $G$ and express $\mathbb{C}^{2^{n}}$ as $V_{1} \oplus V_{2} \ldots \oplus V_{k}$, with $V_{i}$ corresponding to different types of irreducible representations of $G$.

Then, we compose each $f_{\pi}$ with an appropriate $g_{\pi} \in G$. The resulting transformation $f_{\pi}^{\prime}=g_{\pi} f_{\pi}$ still implements the same permutation $\pi$ of $\hat{\mathcal{X}}$ because $g_{\pi}$ maps every $X \in \hat{\mathcal{X}}$ to itself. We can choose the transformations $g_{\pi}$ so that, on every $V_{i}, f_{\pi}^{\prime} f_{\sigma}^{\prime}$ is the same as $f_{\pi \sigma}^{\prime}$ up to a phase $\left(f_{\pi \sigma}^{\prime}=\right.$ $c_{\pi, \sigma, i} f_{\pi}^{\prime} f_{\sigma}^{\prime}$ for some unit $\left.c_{\pi, \sigma, i} \in \mathbb{C}\right)$.

The next step is eliminating the phase factors $c_{\pi, \sigma, i}$. This is done by considering a larger space $V_{1} \otimes V_{1}^{*}+\ldots+V_{k} \otimes V_{k}^{*}$ and transformations $f_{\pi}^{\prime \prime}=f_{\pi}^{\prime} \otimes\left(f_{\pi}^{\prime}\right)^{*}$ on this larger space. Then, the phase factors $c_{\pi, \sigma, i}$ (from $f^{\prime}$ ) and $c_{\pi, \sigma, i}^{*}$ (from $\left.\left(f^{\prime}\right)^{*}\right)$ cancel out and we get $f_{\pi \sigma}^{\prime \prime}=c_{\pi, \sigma, i} c_{\pi, \sigma, i}^{*} f_{\pi}^{\prime \prime} f_{\sigma}^{\prime \prime}=f_{\pi}^{\prime \prime} f_{\sigma}^{\prime \prime}$. Thus, $f_{\pi}^{\prime \prime}$ form a representation of $S_{M}$ on the linear space $V_{1} \otimes V_{1}^{*}+\ldots+V_{k} \otimes V_{k}^{*}$. This representation can be analysed similarly to section , obtaining lower bounds on intersections of invariant subspaces.

\subsection{Representation up to phases $c_{\pi, \sigma, i}$}

Let $G$ be the group of unitary transformations that fix every one of the subspaces $X \in \hat{\mathcal{X}}$.

Then, $\mathbb{C}^{2^{n}}$ is a representation of $G$ and all $h \in \hat{\mathcal{X}}$ are invariant subspaces. (They are fixed by every element of $G$ according to the definition of $G$.) These invariant subspaces decompose into irreducible invariant subspaces.

Consider all the irreducible invariant subspaces of $\mathbb{C}^{2^{n}}$. Split them into equivalence classes consisting of isomorphic irreducible subspaces. Let $E_{1}, \ldots, E_{k}$ be these equivalence classes. Let $V_{1}$ be the subspace of $\mathbb{C}^{2^{n}}$ spanned by all the irreducible subspaces in $E_{1}$ (i.e., the subspace spanned by all the vectors belonging to at least one subspace in $E_{1}$ ). Let $V_{2}, \ldots, V_{k}$ be defined similarly.

Claim 1. If $i, j \in\{1, \ldots, k\}$ and $i \neq j$, then $V_{i} \perp V_{j}$.

Therefore, $\mathbb{C}^{2^{n}}=V_{1} \oplus V_{2} \oplus \ldots \oplus V_{k}$. Next, we show that transformations $f_{\pi}$ map each $V_{i}$ to some (possibly different) $V_{i^{\prime}}$.

Claim 2. Let $V$ be an invariant subspace. Then, $f_{\pi}(V)$ is invariant as well. If $V$ is irreducible, $f_{\pi}(V)$ is irreducible. Moreover, if $V$ and $V^{\prime}$ are two isomorphic irreducible subspaces, $f_{\pi}(V)$ and $f_{\pi}\left(V^{\prime}\right)$ are isomorphic as well.

Proof: The map $g \rightarrow f_{\pi} g f_{\pi}^{-1}$ is an automorphism of $G$. If $V$ is invariant under the action of $g, f_{\pi}(V)$ is invariant under the action of $f_{\pi} g f_{\pi}^{-1}$. Therefore, if $V$ is invariant under $G$, so is $f_{\pi}(V)$.

If $f_{\pi}(V)$ is not irreducible, it decomposes into two or more invariant subspaces: $f_{\pi}(V)=W_{1} \oplus W_{2}$. Then, $f_{\pi}^{-1}\left(W_{1}\right)$ is invariant as well, implying that $V$ is not irreducible.

Finally, let $h: V \rightarrow V^{\prime}$ be a $G$-isomorphism of $V$ and $V^{\prime}$ (an isomorphism that commutes with the action of $G$ ). Let $h^{\prime}: f_{\pi}(V) \rightarrow f_{\pi}\left(V^{\prime}\right)$ be defined by $h^{\prime}=f_{\pi} h f_{\pi}^{-1}$. Then, for any $g=f_{\pi} g^{\prime} f_{\pi}^{-1}$, we have

$$
h^{\prime} g=\left(f_{\pi} h f_{\pi}^{-1}\right)\left(f_{\pi} g^{\prime} f_{\pi}^{-1}\right)=f_{\pi} h g^{\prime} f_{\pi}^{-1}=f_{\pi} g^{\prime} h f_{\pi}^{-1}=g h^{\prime}
$$

and every $g \in G$ can be expressed in the form $f_{\pi} g^{\prime} f_{\pi}^{-1}$. Therefore, $h^{\prime}$ is a $G$-isomorphism of $f_{\pi}(V)$ and $f_{\pi}\left(V^{\prime}\right)$.

Remark. $V$ does not have to be isomorphic to $f_{\pi}(V)$ as a representation of $G$. $f_{\pi}$ establishes the isomorphism of $g$ on $V$ with $f_{\pi} g f_{\pi}^{-1}$ on $f_{\pi}(V)$, but $f_{\pi} g f_{\pi}^{-1}$ does not have to equal $g$ on $f_{\pi}(V)$.

Claim 3. For every $i \in\{1, \ldots, k\}$ there is an $i^{\prime}$ such that $f_{\pi}\left(V_{i}\right)=V_{i^{\prime}}$.

Proof: By Claim 2, every two isomorphic irreducible subspaces get mapped to isomorphic irreducible subspaces. Therefore, all subspaces in $E_{i}$ get mapped to subspaces in the 
same $E_{i^{\prime}}$ and $f_{\pi}\left(V_{i}\right) \subseteq V_{i^{\prime}}$. Similar reasoning applied to $f_{\pi}^{-1}$ implies $f_{\pi}^{-1}\left(V_{i^{\prime}}\right) \subseteq V_{i}$.

For each $i \in\{1, \ldots, k\}, V_{i}$ is the direct sum of some number of isomorphic irreducible subspaces: $V_{i}=V_{i 1} \oplus V_{i 2} \oplus \ldots V_{i j_{i}}$. We fix $G$-isomorphisms $h_{i j j^{\prime}}$ between $V_{i j}$ and $V_{i j^{\prime}}$ so that $h_{i j^{\prime} j^{\prime \prime}} h_{i j j^{\prime}}=h_{i j j^{\prime \prime}}$. (By Schur's lemma, each of these isomorphisms is unique up to a multiplicative constant. The isomorphisms can be made to compose properly by adjusting these constants. Note of course that $h_{i j j}$ is the identity.)

Claim 4. $W \subseteq V_{i}$ is an irreducible invariant subspace if and only if

$$
W=\left\{a_{j} x+a_{j+1} h_{i j(j+1)}(x)+\ldots+a_{j_{i}} h_{i j j_{i}}(x) \mid x \in V_{i j}\right\}
$$

for some $j \in\left\{1, \ldots, j_{i}\right\}$ and $a_{j}, \ldots, a_{j_{i}} \in \mathbb{C}$.

Proof: "If" part:

Invariance:

$$
g\left(\sum_{\ell=j}^{j_{i}} a_{\ell} h_{i j \ell}(x)\right)=\sum_{\ell=j}^{j_{i}} a_{\ell} g\left(h_{i j \ell}(x)\right)=\sum_{\ell=j}^{j_{i}} a_{\ell} h_{i j \ell}(g(x))
$$

because each $h_{i j \ell}$ is a $G$-isomorphism.

$W$ is irreducible because, if $W_{1} \subset W$ and $W_{1}$ is invariant, then

$$
\left\{x \mid a_{j} x+a_{j+1} h_{i j(j+1)}(x)+\ldots+a_{j_{i}} h_{i j j_{i}}(x) \in W_{1}\right\}
$$

is an invariant subspace of $V_{i j}$; but $V_{i j}$ is irreducible and $\operatorname{dim}(W)=\operatorname{dim}\left(V_{i j}\right)$.

"Only if" part:

Let $W$ be an irreducible invariant subspace of $V_{i}$. Let $x^{\prime} \in$ $W$. Then, we can write $x^{\prime}$ as $x_{1}^{\prime}+\ldots+x_{j_{i}}^{\prime}, x_{1}^{\prime} \in V_{i 1}$, $\ldots, x_{j_{i}}^{\prime} \in V_{i j_{i}}$. If $x^{\prime} \neq x^{\prime \prime} \in W$, then for any index $j$, $x_{j}^{\prime} \neq x_{j}^{\prime \prime}$ or $x_{j}^{\prime}=x_{j}^{\prime \prime}=0$. (Otherwise, $W \cap \bigoplus_{\ell \neq j} V_{i \ell}$ is a nontrivial subspace of $W$. It is invariant because $V_{i}$ and $V_{i j}$ are invariant. Contradiction with the irreducibility of $W$.) Let $j$ be the smallest index for which there is an $x^{\prime} \in W$ with $x_{j}^{\prime} \neq 0$. Then, for every $x \in V_{i j}$, there is an $x^{\prime} \in W$ with $x_{j}^{\prime}=x$. (For, if $A$ and $B$ are invariant subspaces of a unitary representation, the projection of $A$ onto $B$ is invariant. Apply this with $A=W$ and $B=V_{i j}$, then use the irreducibility of $V_{i j}$.)

The above considerations allow us to define the mapping $h_{j j^{\prime}}: V_{i j} \rightarrow V_{i j^{\prime}}$ by $h_{j j^{\prime}}\left(x_{j}^{\prime}\right)=x_{j^{\prime}}^{\prime}$. By the definition, $h_{j j^{\prime}}\left(g\left(x_{j}^{\prime}\right)\right)=h_{j j^{\prime}}\left(\left(g\left(x^{\prime}\right)\right)_{j}\right)=\left(g\left(x^{\prime}\right)\right)_{j^{\prime}}=g\left(x_{j^{\prime}}^{\prime}\right)=g\left(h_{j j^{\prime}}\left(x_{j}^{\prime}\right)\right.$, so $h_{j j^{\prime}}$ is a $G$-isomorphism. By Schur's lemma, this implies that $h_{j j^{\prime}}=a_{j^{\prime}} h_{i j j^{\prime}}$ for some $a_{j^{\prime}} \in \mathbb{C}$.

In general, if $W$ is any irreducible invariant subspace of $\mathbb{C}^{2^{n}}$, then $W$ must be in the form described by claim for some $i$. ( $W$ belongs to some equivalence class $E_{i}$ and therefore is contained in the corresponding $V_{i}$.)

For each $V_{i 1}(i \in\{1, \ldots, k\})$, we fix an orthonormal basis $v_{i 1}, \ldots, v_{i t}$. This also fixes a related basis $h_{i 1 j}\left(v_{i 1}\right), \ldots$, $h_{i 1 j}\left(v_{i t}\right)$ for each $V_{i j}$. Moreover, we also get a similar basis

$$
\sum_{\ell=j}^{j_{i}} a_{\ell} h_{i 1 \ell}\left(v_{i 1}\right), \ldots, \sum_{\ell=j}^{j_{i}} a_{\ell} h_{i 1 \ell}\left(v_{i t}\right)
$$

for every invariant irreducible $W \subseteq V_{i}$ because any such $W$ can be written in the form given by claim 4 . We call these bases designated.

This designated basis is exactly the basis for $W$ that can be obtained by applying the isomorphism between $V_{i 1}$ and $W$ to the basis for $V_{i 1}$. Moreover, if $W, W^{\prime}$ are two isomorphic irreducible subspaces, the designated basis for $W$ is mapped to the designated basis for $W^{\prime}$ by the isomorphism between $W$ and $W^{\prime}$.

We are going to impose the following condition on $f_{\pi}^{\prime}$ :

Condition. Let $W$ be an irreducible representation of $G$ and $w_{1}, \ldots, w_{l}$ be the designated basis of $W$. Let $w_{1}^{\prime}, \ldots, w_{l}^{\prime}$ be the designated basis of $f_{\pi}^{\prime}(W)$. Then, there exists $c \in \mathbb{C}$, $|c|=1$ such that $f_{\pi}^{\prime}\left(w_{1}\right)=c w_{1}^{\prime}, \ldots, f_{\pi}^{\prime}\left(w_{l}\right)=c w_{l}^{\prime}$.

Next, we show that this condition suffices to guarantee $f_{\pi}^{\prime} f_{\sigma}^{\prime}=$ $c_{\pi, \sigma, i} f_{\pi \sigma}^{\prime}$ on every $V_{i}$ and that any $f_{\pi}$ that permutes $X \in \hat{\mathcal{X}}$ without satisfying this condition can be transformed into $f_{\pi}^{\prime}$ that satisfies the condition and still permutes the subspaces in the same way.

First, we show that it is enough to ensure that the designated basis of $V_{i 1}$ is mapped correctly for every $i \in\{1, \ldots, k\}$.

Claim 5. Assume that the condition is true for $W=V_{i 1}$. Then, it is also true for any irreducible $W \subseteq V_{i}$.

Proof: Let $h$ be the isomorphism between $V_{i 1}, W$. Note that $h$ maps the designated basis of $V_{i 1}$ to the designated basis of $W$.

Then (by claim 21) $f_{\pi}^{\prime} h\left(f_{\pi}^{\prime}\right)^{-1}$ is an isomorphism between $f_{\pi}^{\prime}\left(V_{i 1}\right)$ and $f_{\pi}^{\prime}(W)$. We know that there is an isomorphism between these two irreducibles that maps the designated basis of one of them to the designated basis of the other. By Schur's lemma, any two isomorphisms of irreducible subspaces can differ only by a multiplicative constant $c$. The unitarity of $f_{\pi}^{\prime} h\left(f_{\pi}^{\prime}\right)^{-1}$ implies that $|c|=1$.

Therefore, $f_{\pi}^{\prime} h\left(f_{\pi}^{\prime}\right)^{-1}$ maps the designated basis of $f_{\pi}^{\prime}\left(V_{i 1}\right)$ to $c$ times the designated basis of $f_{\pi}^{\prime} h\left(f_{\pi}^{\prime}\right)^{-1}\left(f_{\pi}^{\prime}\left(V_{i 1}\right)\right)=$ $f_{\pi}^{\prime} h\left(V_{i 1}\right)=f_{\pi}^{\prime}(W)$. We know that $\left(f_{\pi}^{\prime}\right)^{-1}$ maps the designated basis of $f_{\pi}^{\prime}\left(V_{i 1}\right)$ to the designated basis of $V_{i 1}$ and that $h$ maps the designated basis of $V_{i 1}$ to the designated basis of $W$. This implies that $f_{\pi}^{\prime}$ maps the designated basis of $W$ to $c$ times the designated basis of $f_{\pi}^{\prime}(W)$.

Next, we show how to transform $f_{\pi}$ into $f_{\pi}^{\prime}$ that performs the same permutation $\pi$ of $\hat{\mathcal{X}}$ and maps the designated basis of every $V_{i 1}$ as required.

Let $W_{1}, \ldots, W_{k}$ be $f_{\pi}\left(V_{11}\right), \ldots, f_{\pi}\left(V_{k 1}\right)$. Each of $W_{i}$ lies within one of $V_{1}, \ldots, V_{k}$. Denote this subspace $V_{i^{\prime}}$. Then, for $i \neq j, V_{i^{\prime}} \neq V_{j^{\prime}}$. For each $i \in\{1, \ldots, k\}$, we define a unitary transformation $g_{\pi, i}$ on $V_{i}$ such that $g_{\pi, i^{\prime}} f_{\pi}$ maps the designated basis of $V_{i 1}$ to the designated basis of $f_{\pi}\left(V_{i 1}\right)$. By Claim 4, the irreducible subspace $W_{i}=f_{\pi}\left(V_{i 1}\right)$ is just

$$
\left\{a_{i^{\prime} j} x+a_{i^{\prime}(j+1)} h_{i^{\prime} j(j+1)}(x)+\ldots+a_{i^{\prime} j_{i^{\prime}}} h_{i^{\prime} j j_{i^{\prime}}}(x) \mid x \in V_{i^{\prime} j}\right\}
$$

for some $j$. Moreover, the mapping that maps each $v \in W_{i}$ to its $V_{i^{\prime} j}$-component is an isomorphism of $W_{i}$ and $V_{i^{\prime} j}$ w.r.t. $G$ (similarly to proof of Claim 4 ).

Let $v_{1}, \ldots, v_{l}$ be the designated basis of $V_{i 1}, v_{1}^{\prime}, \ldots, v_{l}^{\prime}$ be $f_{\pi}\left(v_{1}\right), \ldots, f_{\pi}\left(v_{l}\right)$ and $v_{1}^{\prime \prime}, \ldots, v_{l}^{\prime \prime}$ be the $V_{i^{\prime} j}$ components of $v_{1}^{\prime}, \ldots, v_{l}^{\prime}$.

Let $w_{1}, \ldots, w_{l}$ be the designated basis of $V_{i^{\prime} j}$ and $g_{\pi, i^{\prime} j}$ be the unitary transformation on $V_{i^{\prime} j}$ that maps $v_{1}^{\prime \prime}, \ldots, v_{l}^{\prime \prime}$ to $w_{1}, \ldots, w_{l}$. We define a unitary transformation $g_{\pi, i^{\prime} j^{\prime}}$ (for every $j^{\prime} \neq j$ ) on $V_{i^{\prime} j^{\prime}}$ to be $h_{i^{\prime} j j^{\prime}} g_{\pi, i^{\prime} j} h_{i^{\prime} j j^{\prime}}^{-1}$. Finally, we take the transformation $g_{\pi, i^{\prime}}$ of $V_{i^{\prime}}$ that is equal to $g_{\pi, i^{\prime} j}$ on each $V_{i^{\prime} j}$. Then, $g_{\pi, i^{\prime}}$ maps

$$
v_{1}^{\prime}=a_{i^{\prime} j} v_{1}^{\prime \prime}+a_{i^{\prime}(j+1)} h_{i^{\prime} j(j+1)}\left(v_{1}^{\prime \prime}\right)+\ldots+a_{i^{\prime} j_{i^{\prime}}} h_{i^{\prime} j j_{i^{\prime}}}\left(v_{1}^{\prime \prime}\right)
$$


to

$$
\begin{gathered}
a_{i^{\prime} j} g_{\pi, i^{\prime} j}\left(v_{1}^{\prime \prime}\right)+a_{i^{\prime}(j+1)} g_{\pi, i^{\prime}(j+1)} h_{i^{\prime} j(j+1)}\left(v_{1}^{\prime \prime}\right)+\ldots= \\
a_{i^{\prime} j} g_{\pi, i^{\prime} j}\left(v_{1}^{\prime \prime}\right)+a_{i^{\prime}(j+1)} h_{i^{\prime} j(j+1)} g_{\pi, i^{\prime} j}\left(v_{1}^{\prime \prime}\right)+\ldots \\
=a_{i^{\prime} j} w_{1}+a_{i^{\prime}(j+1)} h_{i^{\prime} j(j+1)}\left(w_{1}\right)+\ldots
\end{gathered}
$$

which is exactly the first vector of the designated basis for $W_{i}$. The same is true for $v_{2}^{\prime}, \ldots, v_{l}^{\prime}$, implying that $g_{\pi, i^{\prime}} f_{\pi}$ maps the designated basis of $V_{i 1}$ to the designated basis of $W_{i}$.

Now, we take $g_{\pi}$ that is equal to $g_{\pi, i}$ on each $V_{i}$ and take $f_{\pi}^{\prime}=g_{\pi} f_{\pi}$.

\section{Claim 6. $g_{\pi}$ preserves all $X \in \hat{\mathcal{X}}$.}

Proof: By definition, the restriction $\left.g_{\pi}\right|_{V_{i}}$ is equal to $g_{\pi, i}$, and $g_{\pi, i}$ clearly preserves $V_{i 1}, \ldots, V_{i j_{i}}$. Moreover, $g_{\pi, i}$ (and, hence, $\left.g_{\pi}\right)$ preserves any irreducible subspace $W \subseteq V_{i}$ because any such subspace is in the form of claim 1 .

Every $X \in \hat{\mathcal{X}}$ is invariant under $G$. Therefore, it decomposes into a direct sum of irreducible subspaces. Each of these subspaces is in one of the classes $E_{1}, \ldots, E_{k}$ and, therefore, lies in one of $V_{1}, \ldots, V_{k}$. This means that it is preserved by $g_{\pi}$. Therefore, $X$ which is a direct sum of such irreducible subspaces is preserved by $g_{\pi}$ as well.

Hence, $f_{\pi}^{\prime}=g_{\pi} f_{\pi}$ realizes the same permutation $\pi$ of $X \in \hat{\mathcal{X}}$ as $f_{\pi}$. $\mathbb{C}$.

Claim 7. On every $V_{i}, f_{\pi}^{\prime} f_{\sigma}^{\prime}=c_{\pi, \sigma, i} f_{\pi \sigma}^{\prime}$ for some $c_{\pi, \sigma, i} \in$

Proof: This is equivalent to showing that $\left(f_{\pi \sigma}^{\prime}\right)^{-1} f_{\pi}^{\prime} f_{\sigma}^{\prime}$ is equal to $c_{\pi, \sigma, i}$ times the identity. To show that, notice that $\left(f_{\pi \sigma}^{\prime}\right)^{-1} f_{\pi}^{\prime} f_{\sigma}^{\prime}$ maps every subspace $X \in \hat{\mathcal{X}}$ to itself because $\left(f_{\pi \sigma}^{\prime}\right)^{-1}$ performs the inverse of the permutation $\pi \sigma$ on $\hat{\mathcal{X}}$. Therefore, $\left(f_{\pi \sigma}^{\prime}\right)^{-1} f_{\pi}^{\prime} f_{\sigma}^{\prime} \in G$. This means that $V_{i j}$ are all preserved by $\left(f_{\pi \sigma}^{\prime}\right)^{-1} f_{\pi}^{\prime} f_{\sigma}^{\prime}$.

Moreover, $f_{\sigma}^{\prime}, f_{\pi}^{\prime}$ and $f_{\pi \sigma}^{-1}$ all map the designated bases to $c$ times designated bases (Claim 5). Therefore, $\left(f_{\pi \sigma}^{\prime}\right)^{-1} f_{\pi}^{\prime} f_{\sigma}^{\prime}$ maps the designated basis of $V_{i j}$ to $c$ times the designated basis of $\left(f_{\pi \sigma}^{\prime}\right)^{-1} f_{\pi}^{\prime} f_{\sigma}^{\prime}\left(V_{i j}\right)=V_{i j}$.

It remains to show that $c$ is the same for all irreducible subspaces $V_{i j}$ contained in $V_{i}$. Let $c_{j}$ and $c_{j^{\prime}}$ be the values of $c$ for $V_{i j}$ and $V_{i j^{\prime}}$. Consider the subspace

$$
W=\left\{x+h_{i j j^{\prime}}(x) \mid x \in V_{i j}\right\} .
$$

By Claim 1 , this is an irreducible invariant subspace. Now, $\left(f_{\pi \sigma}^{\prime}\right)^{-1} f_{\pi}^{\prime} f_{\sigma}^{\prime}$ maps it to

$$
\begin{gathered}
W^{\prime}=\left\{c_{j} x+c_{j^{\prime}} h_{i j j^{\prime}}(x) \mid x \in V_{i j}\right\}= \\
\left\{x+\frac{c_{j}}{c_{j^{\prime}}} h_{i j j^{\prime}}(x) \mid x \in V_{i j}\right\} .
\end{gathered}
$$

The invariance of $W$ means that $W^{\prime}=W$ and $c_{j}=c_{j^{\prime}}$. Therefore, $c_{j}$ are all equal. This means that $\left(f_{\pi \sigma}^{\prime}\right)^{-1}(x)=$ $c_{j} x$ for all $x \in V_{i}$ because the designated bases of $V_{i j}$ together form a basis for entire subspace $V_{i}$.

Unfortunately, arguments of this type (composing $f_{\pi}$ with an appropriate transformation that fixes all $U_{i}$ ) cannot be used to eliminate phases $c_{\pi, \sigma, i}$.

The reason for this is that there exist so-called projective representations. A projective representation is a set of maps $f_{\pi}$ such that $f_{\pi} f_{\sigma}=c_{\pi, \sigma} f_{\pi \sigma}, c_{\pi, \sigma} \in \mathbb{C}$. It is known that the symmetric group has projective representations which are not equivalent to any of the usual representations 6 .

One possible solution would be to use the standard forms of projective representations which are quite well studied 6 . However, to be able to use them, we would need to show that the multiplicative constants $c_{\pi, \sigma, i}$ are the same for all $V_{i}$ (or show that we can split all $V_{i}$ in several groups so that $c_{\pi, \sigma, i}$ is the same within one group) and we do not know if this is possible.

Our solution is to replace $f_{\pi}^{\prime}$ by transformations $f_{\pi}^{\prime \prime}$ on a larger space $V_{1} \otimes V_{1}^{*}+\ldots V_{k} \otimes V_{k}^{*}$ so that $f_{\pi}^{\prime \prime} f_{\sigma}^{\prime \prime}=f_{\pi \sigma}^{\prime \prime}$. Then, $f_{\pi}^{\prime \prime}$ form a representation in the usual sense and we can analyse them similarly to section 1 .

\subsection{Solving the problem with phases}

We split $V_{1}, \ldots, V_{k}$ into equivalence classes $\mathcal{V}_{1}, \ldots \mathcal{V}_{l} . V_{i}$ and $V_{j}$ are in one class if there is a $\pi \in S_{M}$ such that $f_{\pi}\left(V_{i}\right)=V_{j}$. Let $W_{i}$ be the union of all $V_{j}$ that belong to $\mathcal{V}_{i}$. Then, $f_{\pi}\left(W_{i}\right)=W_{i}$ for any $\pi \in S_{M}$ (because $f_{\pi}$ maps every $V_{j} \in$ $\mathcal{V}_{i}$ to some $\left.V_{j^{\prime}} \in \mathcal{V}_{i}\right)$. Therefore, we can look at each $W_{i}$ separately.

Lemma 7. Let $X, Y \in \hat{\mathcal{X}}$. Then, for any $t$,

$$
\operatorname{dim} X \cap W_{t}-\operatorname{dim} X \cap Y \cap W_{t} \leq \sqrt{\frac{4 c n}{M}} \operatorname{dim} W_{t} .
$$

Proof: To simplify the notation, assume that $W_{t}=V_{1} \oplus$ $V_{2} \ldots \oplus V_{l}$.

Consider the linear space $W_{t}^{\prime}=V_{1} \otimes V_{1}^{*} \oplus \ldots V_{l} \otimes V_{l}^{*}$ and the linear transformations $f_{\pi}^{\prime \prime}=f_{\pi}^{\prime} \otimes f_{\pi}$. These linear transformations form a representation because

$$
\begin{gathered}
f_{\pi \sigma}^{\prime} \otimes\left(f_{\pi \sigma}^{\prime}\right)^{*}=c_{\pi, \sigma, i} f_{\pi}^{\prime} f_{\sigma}^{\prime} \otimes c_{\pi, \sigma, i}^{*}\left(f_{\pi}^{\prime}\right)^{*}\left(f_{\sigma}^{\prime}\right)^{*}= \\
f_{\pi}^{\prime} f_{\sigma}^{\prime} \otimes\left(f_{\pi}^{\prime}\right)^{*}\left(f_{\sigma}^{\prime}\right)^{*}=f_{\pi}^{\prime \prime} f_{\sigma}^{\prime \prime}
\end{gathered}
$$

on every $V_{i} \otimes V_{i}^{*}$.

Let

$$
X^{\prime}=\oplus_{i=1}^{l}\left(X \cap V_{i}\right) \otimes\left(X \cap V_{i}\right)^{*}
$$

be the subspace of $W_{t}^{*}$ corresponding to $X$. Then, $f_{\pi}^{\prime}(X)=$ $Y$ implies $f_{\pi}^{\prime \prime}\left(X^{\prime}\right)=Y^{\prime}$. (To see this, consider one of $(X \cap$ $\left.V_{i}\right) \otimes\left(X \cap V_{i}\right)^{*}$. Assume that $f_{\pi}^{\prime}$ maps $V_{i}$ to $V_{i^{\prime}}$. Then, $f_{\pi}^{\prime}(X)=Y$ implies $f_{\pi}^{\prime}\left(X \cap V_{i}\right)=Y \cap V_{i^{\prime}}$ and

$$
f_{\pi}^{\prime \prime}\left(\left(X \cap V_{i}\right) \otimes\left(X \cap V_{i}\right)^{*}\right)=\left(Y \cap V_{i^{\prime}}\right) \otimes\left(Y \cap V_{i^{\prime}}\right)^{*} .
$$

Combining these equalities for all $V_{i}$ gives $f_{\pi}^{\prime \prime}\left(X^{\prime}\right)=Y^{\prime}$.) In particular, $f_{\pi}^{\prime \prime}\left(X^{\prime}\right)=Y^{\prime}$ means that $X^{\prime}$ is invariant under all $\pi \in S_{M}$ satisfying $\pi(X)=X$. Therefore, by Lemma 6 ,

$$
\operatorname{dim} X^{\prime}-\operatorname{dim} X^{\prime} \cap Y^{\prime} \leq \frac{4 c n}{M} \operatorname{dim} W_{t}^{\prime} .
$$

We use this inequality to derive a bound on $\operatorname{dim} X \cap W_{t}-$ $\operatorname{dim} X \cap Y \cap W_{t}$. To do this, we relate the dimensions of $X \cap V_{i}$ and $X^{\prime} \cap\left(V_{i} \otimes V_{i}^{*}\right)$. First, notice that we have

$$
X \cap W_{t}=\oplus_{i=1}^{l}\left(X \cap V_{i}\right)
$$

because $X$ is invariant under $G$ and, therefore, can be written as a sum of irreducible invariant subspaces (and each of these irreducibles is contained in some $V_{i}$ ). The same is true about $Y$ and $X \cap Y$ :

$$
X \cap Y \cap W_{t}=\oplus_{i=1}^{l}\left(X \cap Y \cap V_{i}\right)
$$


Let $d_{i}$ and $d_{i}^{\prime}$ be the dimensions of $X \cap V_{i}$ and $X \cap Y \cap$ $V_{i}$. Then, (2) and (3) imply that $\operatorname{dim} X \cap W_{t}=\sum_{i=1}^{l} d_{i}$, $\operatorname{dim} X \cap Y \cap W_{t}=\sum_{i=1}^{l} d_{i}^{\prime}$ and

$$
\operatorname{dim} X \cap W_{t}-\operatorname{dim} X \cap Y \cap W_{t}=\sum_{i=1}^{l}\left(d_{i}-d_{i}^{\prime}\right) .
$$

If we look at $V_{i} \otimes V_{i}^{*}$, then

$$
X^{\prime} \cap\left(V_{i} \otimes V_{i}^{*}\right)=\left(X \cap V_{i}\right) \otimes\left(X \cap V_{i}\right)^{*} .
$$

This implies $\operatorname{dim} X^{\prime} \cap\left(V_{i} \otimes V_{i}^{*}\right)=d_{i}^{2}$ and $\operatorname{dim} X^{\prime}=\sum_{i} d_{i}^{2}$. Similarly, $\operatorname{dim} X^{\prime} \cap Y^{\prime}=\sum_{i=1}^{l}{d_{i}^{\prime}}^{2}$.

Let $d$ be the dimension of $V_{1}$. Then, the dimensions of $V_{2}$, $\ldots, V_{l}$ are $d$ as well because, for every $i \in\{2, \ldots, l\}$, there is a unitary $f_{\pi}$ such that $f_{\pi}\left(V_{1}\right)=V_{i}$. Therefore, $\operatorname{dim} W_{1}=l d$. Also, $\operatorname{dim} W_{t}^{\prime}=l d^{2}$ because $\operatorname{dim} V_{i} \otimes V_{i}^{*}=d^{2}$ for every $i \in\{1, \ldots, l\}$. Hence, we have

$$
\begin{gathered}
\frac{\operatorname{dim} X \cap W_{t}-\operatorname{dim} X \cap Y \cap W_{t}}{\operatorname{dim} W_{t}}=\frac{\sum_{i=1}^{m}\left(d_{i}-d_{i}\right)}{m d}= \\
\frac{1}{m} \sum_{i=1}^{m} \sqrt{\frac{\left(d_{i}-d_{i}^{\prime}\right)^{2}}{d^{2}}} \leq \frac{1}{m} \sum_{i=1}^{m} \sqrt{\frac{\left(d_{i}-d_{i}^{\prime}\right)\left(d_{i}+d_{i}^{\prime}\right)}{d^{2}}}= \\
\frac{1}{m} \sum_{i=1}^{m} \sqrt{\frac{\left(d_{i}^{2}-d_{i}^{\prime 2}\right)}{d^{2}}} .
\end{gathered}
$$

Convexity of the square root implies that this is at most

$$
\sqrt{\frac{\sum_{i=1}^{m}\left(d_{i}^{2}-d_{i}^{\prime 2}\right)}{m d^{2}}}=\sqrt{\frac{\operatorname{dim} X^{\prime}-\operatorname{dim} X^{\prime} \cap Y^{\prime}}{\operatorname{dim} W_{t}^{\prime}}} .
$$

Equation 1 implies that this is at most $\sqrt{(4 c n) / M}$. This completes the proof of lemma.

With Lemma 7, we can finish the proof similarly to the simple case (section 4). By summing over $W_{t}$ 's, we get

$$
\operatorname{dim} X-\operatorname{dim} X \cap Y \leq \sqrt{\frac{2 c n}{M}} \sum_{t} \operatorname{dim} W_{t}=\sqrt{\frac{2 c n}{M}} 2^{n} .
$$

Therefore,

$$
\frac{\operatorname{dim} X \cap Y}{\operatorname{dim} X} \geq \frac{2^{n-k}-\sqrt{\frac{2 c n}{M}} 2^{n}}{2^{n-k}}=1-\frac{2^{k+1} \sqrt{c n}}{\sqrt{M}} .
$$

If $\frac{2^{k+1} \sqrt{c n}}{\sqrt{M}} \geq \frac{1}{\operatorname{poly}(m)}$, then $m=(2 k+\log n)(1+o(1))$. This completes the proof of Theorem $\mathrm{A}$.

\section{REFERENCES}

[1] Barrington, D. A, "Bounded-width polynomial-size branching programs recognize exactly those languages in NC1", J. Computer and System Sciences 38(1):150164, February 1989.

[2] Chuang, I. L., Vandersypen, L. M. K., Zhou, X., Leung D. W. and Lloyd, S., "Experimental realization of a quantum algorithm", Nature 393, 143-146, 1998.

[3] Cory, D. G., Fahmy, A. F. and Havel, T. F., "Ensemble Quantum Computing by Nuclear Magnetic Resonance Spectroscopy", Proc. Natl. Acad. Sci. 94:16341639, 1997.
[4] Fulton, W. and Harris, J., "Representation Theory. A First Course". Springer-Verlag, 1991.

[5] Gershenfeld, N. and Chuang, I., Science 275, pp. 350, 1997.

[6] Hoffman, P. N. and Humphreys, J. F. "Projective Representations of the Symmetric Groups". Oxford University Press, 1992.

[7] Knill, E. and Laflamme, R., "On the power of one bit of quantum information", Phys. Rev. Lett. 81:5672, 1998.

[8] Mishchenko, S. P. "Lower bounds on the dimensions of irreducible representations of symmetric groups and of the exponents of the exponential of varieties of Lie algebras", Matematicheskiu Sbornik, 187:83-94, 1996.

[9] Nielsen, M., "Probability distributions consistent with a mixed state", LANL archive http://xxx.lanl.gov/abs/quant-ph/9909020.

[10] Rasala, R., On the minimal degrees of characters of $S_{n}$. Journal of Algebra, 45:132-181, 1977.

[11] Schulman, L. J. and Vazirani, U. V., "Molecular Scale Heat Engines and Scalable Quantum Computation", Proc. 31'st Ann. ACM Symp. Theory Comput. 1999.

\section{REPRESENTATION THEORY}

Representation. A representation $\rho$ of a group $G$ is a homomorphism $\rho$ from $G$ to the group of linear transformations $G L(V)$ of a vector space $V$. This means that, for any $g, h \in G, \rho(g h)=\rho(g) \rho(h)$. If the mapping $\rho$ is clear from the context, we often call the space $V$ itself representation of $G$.

Irreducibility. We say that a subspace $W$ is an invariant subspace of a representation $\rho$ if $\rho(g) W \subseteq W$ for all $g \in G$. In order for $W$ to be an invariant subspace for $\rho$, it must be simultanously fixed under all $\rho(g)$. The zero subspace and the subspace $V$ are always invariant. If no nonzero proper subspaces are invariant, the representation is said to be irreducible.

Isomorphism. Two representations $\rho: G \rightarrow G L(V)$ and $\rho^{\prime}: G \rightarrow G L(W)$ are isomorphic if there is a bijective linear map $\varphi: V \rightarrow W$ such that $\varphi \rho(g)=\rho^{\prime}(g) \varphi$ for any $g \in G$.

Schur's Lemma. If $\rho$ and $\rho^{\prime}$ are two irreducible representations and $\varphi$ is an isomorphism between them, then any other isomorphism $\varphi^{\prime}$ between $\rho$ and $\rho^{\prime}$ is $c \varphi$ for some constant $c \in \mathbb{C}$.

Schur's lemma is usually stated for finite groups. However, if the representation is unitary (as in this paper), it is also true for infinite groups.

Decomposition. When a representation does have a nonzero proper invariant subspace $V_{1} \subset V$, it is always possible to find a complementary subspace $V_{2}$ (so that $\left.V=V_{1} \oplus V_{2}\right)$ which is also invariant. Since $\rho(g)$ fixes $V_{1}$, we may let $\rho_{1}(g)$ be the linear map on $V_{1}$ given by $\rho(g)$. It is not hard to see that $\rho_{1}: G \rightarrow G L\left(V_{1}\right)$ is in fact a representation. Similarly define $\rho_{2}(g)$ to be $\rho(g)$ restricted to $V_{2}$. Since $V=V_{1} \oplus V_{2}$, the linear map $\rho(g)$ is completely determined by $\rho_{1}(g)$ and $\rho_{2}(g)$, and in this case we write $\rho=\rho_{1} \oplus \rho_{2}$. 


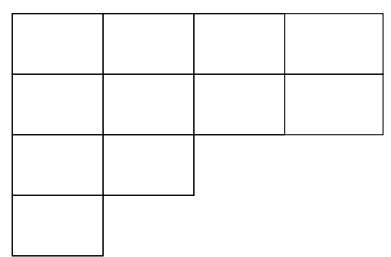

Figure 1: The Young diagram of $\lambda=(4,4,2,1)$.

\begin{tabular}{|l|l|l|l|}
\hline 7 & 5 & 3 & 2 \\
\hline 6 & 4 & 2 & 1 \\
\hline 3 & 1 & \multicolumn{2}{|l}{} \\
\cline { 1 - 2 } 1 & \multicolumn{2}{|l}{} \\
\cline { 1 - 2 } & &
\end{tabular}

Figure 2: The hook-lengths for (4, 4, 2, 1).

Complete Reducibility. Repeating the process described above, a representation $\rho$ may be written $\rho=\rho_{1} \oplus \rho_{2} \oplus$ $\ldots \oplus \rho_{k}$, where each $\rho_{i}$ is irreducible.

Irreducible representations of $S_{M}$. In this paper, we use representations of the symmetric group $S_{M}$. The irreducible representations of $S_{M}$ may be placed into oneto-one correspondence with the partitions of $n$. A partition of $M$ is a sequence $\left(\lambda_{1}, \ldots, \lambda_{k}\right)$ of positive integers, with $\lambda_{1} \geq \ldots \geq \lambda_{k}$ for which $\sum \lambda_{i}=M$. It is customary to identify the partition $\lambda=\left(\lambda_{1}, \ldots, \lambda_{k}\right)$ with a diagram consisting of $k$ rows of boxes, the $i$ th row containing $\lambda_{i}$ boxes. We will let $\lambda$ stand for both the partition and the associated diagram. For example, the diagram corresponding to the partition $\lambda=(4,4,2,1)$ is shown in figure 7 .

The irreducible representation associated with $\lambda$ is denoted $\rho_{\lambda}$. There is an explicit formula for the dimension of $\rho_{\lambda}$. This involves the notion of a hook: for a cell $(i, j)$ of a Young tableau $\lambda$, the $(i, j)$-hook $h_{i, j}$ is the collection of all cells of $\lambda$ which are beneath $(i, j)$ (but in the same column) or to the right of $(i, j)$ (but in the same row), including the cell $(i, j)$. The length of the hook $\ell(h)$ is the number of cells appearing in the hook. With this notation, the dimension of $\rho_{\lambda}$ may be expressed:

$$
\operatorname{dim} \rho_{\lambda}=\frac{n !}{\prod_{i, j} \ell\left(h_{i, j}\right)},
$$

this product being taken over all hooks $h$ of $\lambda$. Figure 7 shows the hook lengths for the partition $\lambda=(4,4,3,1)$. Formula (4) implies that the dimension of corresponding representation is

$$
\frac{11 !}{7 \cdot 5 \cdot 3 \cdot 2 \cdot 6 \cdot 4 \cdot 2 \cdot 3}=1320 \text {. }
$$

Restriction. A representation $\rho$ of a group $G$ is also automatically a representation of any subgroup $H$. Note that even if a representation is irreducible over $G$, it may no longer be irreducible when restricted to $H$.
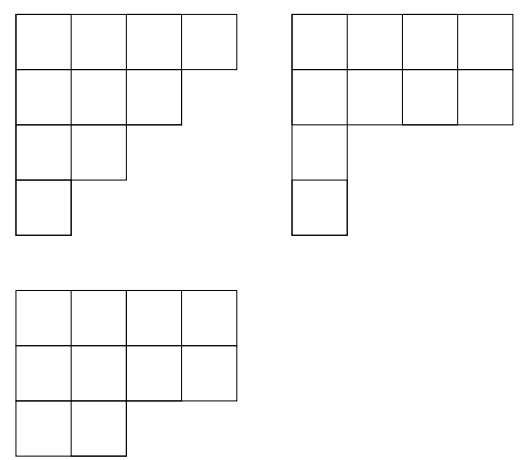

Figure 3: The Young diagrams of representations of $S_{10}$ contained in $\rho_{\lambda}$.

Restriction from $S_{M}$ to $S_{M-1}$. In particular, we will be considering the restrictions of irreducible representations of $S_{M}$ to $S_{M-1}$. Let $\lambda$ be a partition of $M$ and $\rho_{\lambda}$ be the corresponding irreducible representation. Then, when we restrict to $S_{M-1}, \rho_{\lambda}$ decomposes into irreducible representations of $S_{M-1}$ in the following way:

$$
\rho=\bigoplus_{\lambda^{-}} \rho_{\lambda^{-}}
$$

where $\lambda^{-}$ranges over all shapes of size $M-1$ that can be obtained by deleting an "inside corner" from $\lambda$. (An inside corner is simply a point of the shape whose deletion leaves a legal shape.)

For example, the representation $\rho_{\lambda}, \lambda=(4,4,2,1)$ of $S_{11}$ decomposes into 3 irreducible representations of $S_{10}$. The Young diagrams of these representations are shown in Fig. 7 